\title{
Development and Implementation of a New Dynamic Face Detection Operator
}

\author{
B.Abbadi \\ MSE Laboratory \\ University of BBArreridj \\ BP 64 - 34030 - ALGERIA
}

\author{
A.Oulefki \\ MSE Laboratory \\ University of BBArreridj \\ BP 64 - 34030 - ALGERIA
}

\author{
M.Mostefai \\ MSE Laboratory \\ University of BBArreridj \\ BP 64 - 34030 - ALGERIA
}

\begin{abstract}
This work is part of a project dedicated to the development of a real-time multimodal biometric identification system. In this kind of applications, face detection is an important step before any identification process. This paper presents the development and the implementation of a dynamic face detector based on both skin color and motion detection techniques. Our choice will be guided by the computational complexity and timing constraints for a real-time processing on a low cost Virtex FPGA platform.
\end{abstract}

\section{General Terms}

Real time systems - Biometric identification - Algorithms development and implementation

\section{Keywords}

Motion detection - Skin color detection - Face tracking Hardware implementation - FPGA.

\section{INTRODUCTION}

Face detection plays an important role in many applications such as video surveillance, human computer interface, face recognition, etc. Various methods based on Neural Networks (NN) [1], Support Vector Machines (SVM) [2], Hidden Machine Markov (HMM) [3] principal component analysis (PCA), Hough transform (HT), geometrical template matching (GTM) have been developed to perform this task. These methods provide good results, but require complex computation tasks (such as matrix multiplication, eigenvectors computation, square roots, etc) which consume a lot of hardware resources [4].

Another family of local methods based on the exploitation of low level image information (such as forms, intensity, color and motion) is fairly good and requires reasonable hardware resource, but remains less effective in case of varying illumination and complex backgrounds [5].

This paper intends to present an efficient combination of static and dynamic images properties to build a robust real-time face detection module based on both skin, color and motion detection techniques. The developed module will be a part of a complete interactive multimodal biometric identification system.

After an overview of interactive multimodal biometric identification systems, as well as their timing constraints, the approach for the construction of an efficient real-time dynamic face detection module will be presented. The operator is validated by simulation on a standard PC platform and implemented on a Virtex FPGA chip for a real-time processing.

\section{INTERACTIVE BIOMETRIC IDENTIFICATION SYSTEMS}

Generally, classical multimodal biometric identification systems requires dedicated persons to proceed (before any authentication) to the extraction of the physiological or behavioral characteristics of a person. This constraining approach can be avoided by equipping identification systems with virtual character modules capable of interacting and exchanging information with the person to be identified.

In order to allow a natural behavior of the character, face detection and tracking task must be efficiently executed to allow a smooth interaction between the person to be identified and the character. Many works dealing with this problem have been presented in the literature. Most of them were based on the Viola and Jones method [6], which consists of a combination of classifiers trained by AdaBoost algorithm to localize objects of interest in natural scenes. However, the integral image generation (needed by this method) requires important computation tasks and is associated with a long latency delay [7]. A solution to this problem was proposed by Mustaphah in [8]. It consists in reducing the region of interest (ROI) to only the moving skin region by using background subtraction and skin color detection. Unfortunately, the processing time required by the square patch feature classification function is very important (it can reach 700 clock cycles in the case of a face window).

Several research works have also presented the hardware implementation of the face detection operator. M. Sadri et al designed and implemented neural network face detection on FPGA [9]. However, irregular computations were performed using internal PowerPC embedded on the FPGA. Other interesting hardware solutions have been developed. Most of them use specialized processors [10] or dedicated ASICs [11] to perform required irregular and complex computation tasks. In this work it will be shown that it is possible to perform efficient and realistic human face detection by considering this last as a dynamic region of interest. It means that there is a difference between a static face (such as a photograph) and a dynamic one, and also there is a difference between moving skin color regions (such as hands) and moving faces. This feature will allow the character to behave naturally during its interaction with the person to be identified. Thus, it is important to focus on the motion of the mouth and the eyes which have the characteristic to be completely surrounded by skin.

\section{PROPOSED DYNAMIC FACE DETECTION ALGORITHM}

The proposed face detection algorithm allows the extraction of moving pixels surrounded by skin color regions. To do this, a parallel motion and ROI skin color detection are performed. They are followed by a bimodal classification in order to extract only moving pixels within a face (see figure 1). These three modules are detailed as the following: 


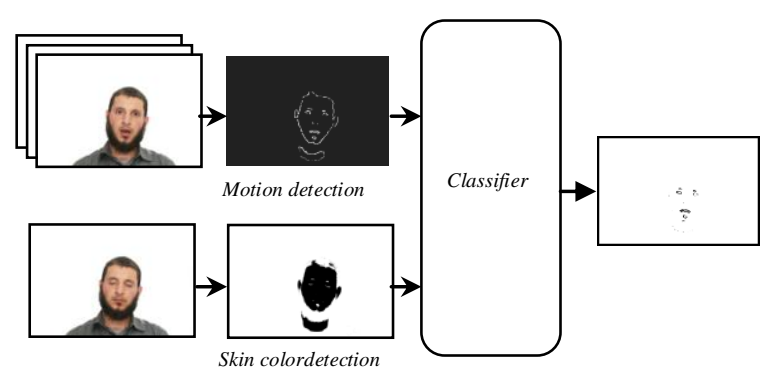

Fig 1: Proposed face detection scheme

\subsection{Motion detection}

Several research works have treated the motion detection task in case of real world scenes. If optical flow methods are excluded (computationally complex and very sensitive to noise) [12], there are those that use background subtraction in their processing and those that exploit varying frontiers between neighbouring images. The two last approaches are well suited to a real-time implementation. However, methods based on background subtraction present some limitations especially in case of scenes with non- controlled lighting. In this case, a differential method has been used, based on Orkisz operator [13]. This last allows the extraction of mobile frontiers by comparing frontiers of the current image to those of next and preceding images. The formulation of this operator is as follow:

$$
\mathrm{H}(\mathrm{x}, \mathrm{y})=\operatorname{Max}\left(\mathrm{G}_{\mathrm{n}-1}, \mathrm{G}_{\mathrm{n}}, \mathrm{G}_{\mathrm{n}+1}\right)-\operatorname{Max}\left(\mathrm{G}_{\mathrm{n}-1}, \mathrm{G}_{\mathrm{n}+1}\right)
$$

with $\mathrm{H}(\mathrm{x}, \mathrm{y})$ the motion amplitude at a position $(\mathrm{x}, \mathrm{y})$ and $\mathrm{G}_{\mathrm{n}-1}, \mathrm{G}_{\mathrm{n}}, \mathrm{G}_{\mathrm{n}+1}$ the respective gradients of three consecutive images $I_{n-1}, I_{n}$ and $I_{n+1}$. An example of results obtained with this operator is presented in figure 2 .

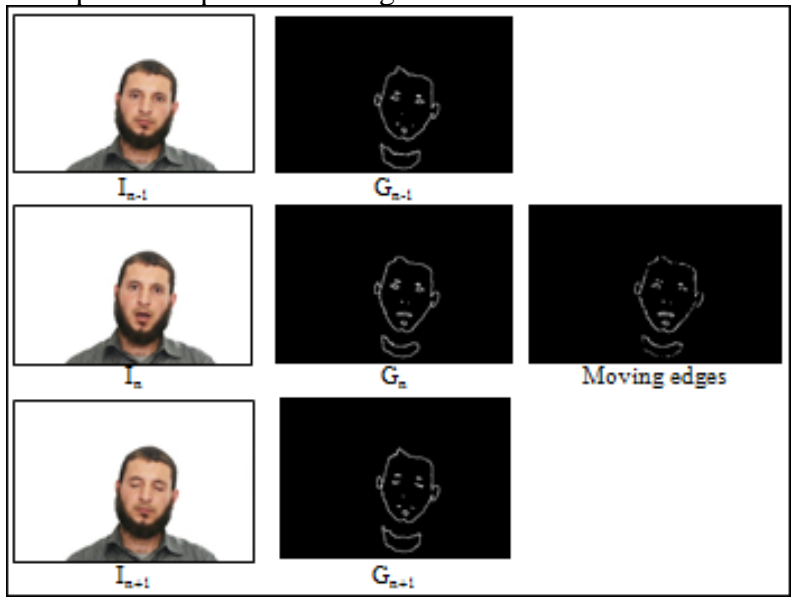

Fig 2: Motion detection with Orkisz operator

To reduce the computation tasks and to allow robust dynamic face detection, only moving pixels which are at least neighbor of two moving pixels are retained for the classification process. An example of selected moving pixels is presented in (figure 3).

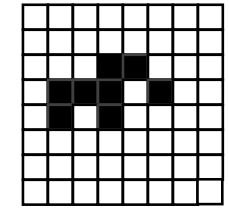

(a) Group of moving pixels

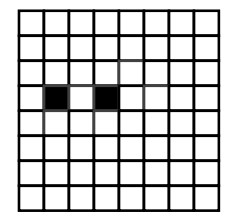

(b) Selected moving pixels
Fig 3: Example of moving pixels selection

\subsection{Skin color detection}

Many different methods have been developed to detect skin regions in a chosen color space. Menser et al. [14] presented a detailed study regarding the choice of the color space according to the application requirements and computation constraints. Acceptable results were obtained with simple color space skin detection methods [15]. However, adaptive color space switching methods seems to be necessary in case of scenes with varying lighting. As a primary work and in order to validate the bimodal face detection approach the following explicit skin detection algorithm has been used:

$$
\begin{gathered}
\text { A pixel with }(R, G, B) \text { values is classified as skin if } \\
\mathrm{R}>95 \& \mathrm{G}>40 \& \mathrm{~B}>20 \& \\
\max \{\mathrm{R}, \mathrm{G}, \mathrm{B}\}-\min \{\mathrm{R}, \mathrm{G}, \mathrm{B}\}>15 \& \\
|\mathrm{R}-\mathrm{G}|>15 \& \mathrm{R}>\mathrm{G} \& \mathrm{R}>\mathrm{B} .
\end{gathered}
$$

This operator helps efficiently to delimitate skin color regions under controlled lighting conditions. But any change in lighting affects considerably detection results. This is due to the chosen skin detection method and not to the proposed approach. An example of results is presented in figure 4 .

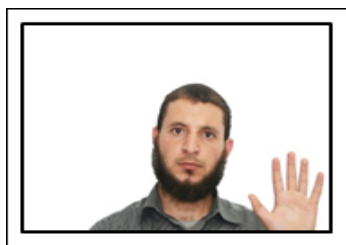

(a) Selected frame

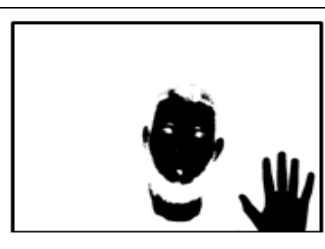

(b) Skin color detection
Fig 4: Skin color detection

\subsection{Classification module}

The classification of the whole selected moving pixels on face or non-face moving pixels is done using a progressive test procedure which allows to check if the selected moving pixel is completely surrounded by skin or not. The test procedure operates in 8 directions as shown in figure 5. Eight variables $\left(\mathrm{Ck}_{0}\right.$ to $\left.\mathrm{Ck}_{7}\right)$ are respectively affected to the eight directions $\left(\operatorname{Dir}_{0}\right.$ to $\left.\operatorname{Dir}_{7}\right)$. To verify that a moving pixel is completely surrounded by skin, all of the eight variables should be set to one. Otherwise the considered moving pixel is erased. Figure 6 presents an example of a classification of two moving pixels on face and non-face moving pixels.

\section{EXPERIMENTAL RESULTS}

Developed face detection method has been validated on a standard PC platform. Experiments were carried out with a great number of persons at different positions from the camera in order to determine a threshold of the face/image surface ratio below which the face detection becomes difficult. Generally, good detection is obtained with a ratio greater than $1 / 15$.

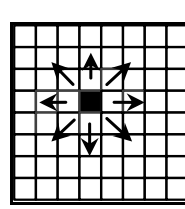

(a)

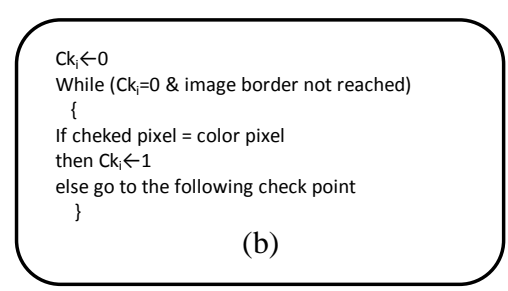

(b) (a) searching directions (b) searching procedure

Fig 5: Classification task 

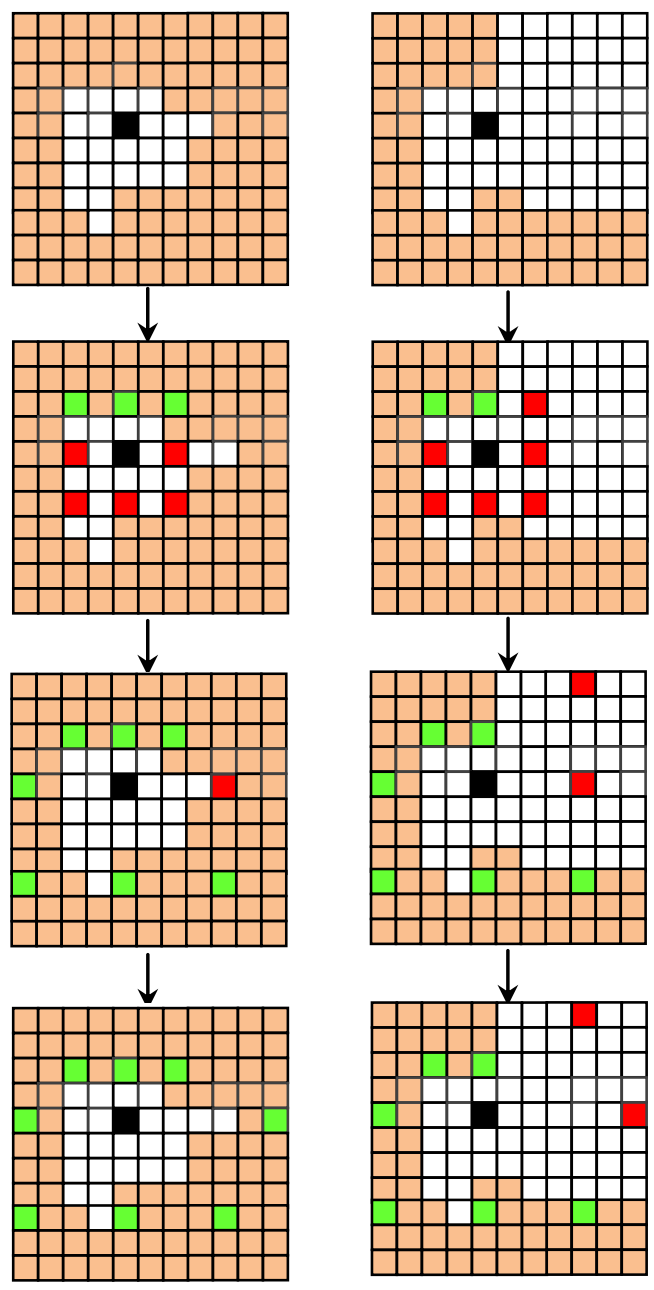

(a) face moving pixel

\begin{tabular}{|l|l|l|l|l|l|}
\hline$\square$ & $\begin{array}{l}\text { Skin Color } \\
\text { pixel }\end{array}$ & $\square$ & $\begin{array}{l}\text { Absence of a } \\
\text { skin color pixel }\end{array}$ & $\mathbf{\square}$ & $\begin{array}{l}\text { A candidate } \\
\text { moving pixel }\end{array}$ \\
\hline$\square$ & $\begin{array}{l}\text { Non skin color } \\
\text { pixel }\end{array}$ & $\square$ & $\begin{array}{l}\text { detection of a } \\
\text { skin color pixel }\end{array}$ & & \\
\hline
\end{tabular}

Fig 6: Example of classification

To confirm that detection concerns only dynamic faces, the filmed person were asked to speak and to blink his/her eyes while making signs with hand (figure 7.a). As shown in figure 7.b, only mouth and eyes parts are retained. Figure $7 \mathrm{c}$ and $7 d$, show the results of the detection of a person filmed beside a photo presenting a human portrait. As detection concerns only the dynamic parts, only the face of the filmed person is localized. Finally, a last test is performed in order to show that the localization can be targeted in the case of the presence of several peoples. In figure $7 \mathrm{e}$ two peoples are facing the camera; one speaks in order to be identified and the other waiting. Automatically the localization targets only the person who is speaking (see figure 7f). This property will allow a natural behavior of the character on the person who speaks.

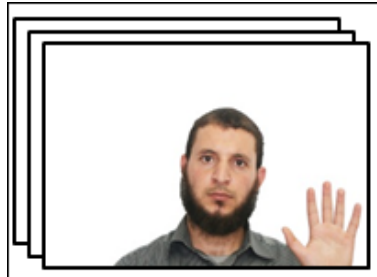

(a) Dynamic face with moving hand

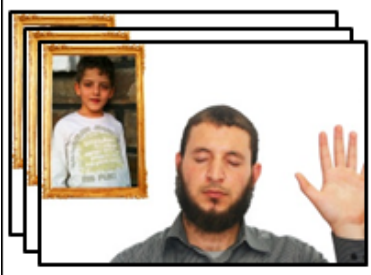

(c) Dynamic face with moving hand and fixed human portrait

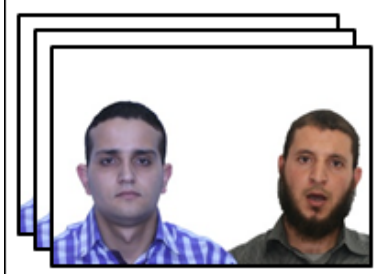

(e) Dynamic face with a static human face

Fig 7: Simulation tests

It is noted that even when respecting the face/image surface ratio, the face detection could fail especially in case of scenes with uncontrolled lighting. A uniform lighting of the scene as well as a smoothing operation of acquired images is therefore necessary if robust and precise face detection is desired.

\section{PROPOSED ARCHITECTURE}

The developed face detection operator is a part of a complete interactive multimodal biometric identification system (see figure 8). This last is based on the following three modules:

- A skin color detection module which extracts regions with skin color ;

- A motion detection module which extracts moving pixels;

- A classifier module which selects moving pixels completely surrounded by skin.

Reconfigurable technology and more particularly Xilinx FPGA technology has been used for the hardware prototyping of the developed operator. The benefits of this last (well known by the whole scientific community working in this field) are: quick and low cost prototyping [16]. 


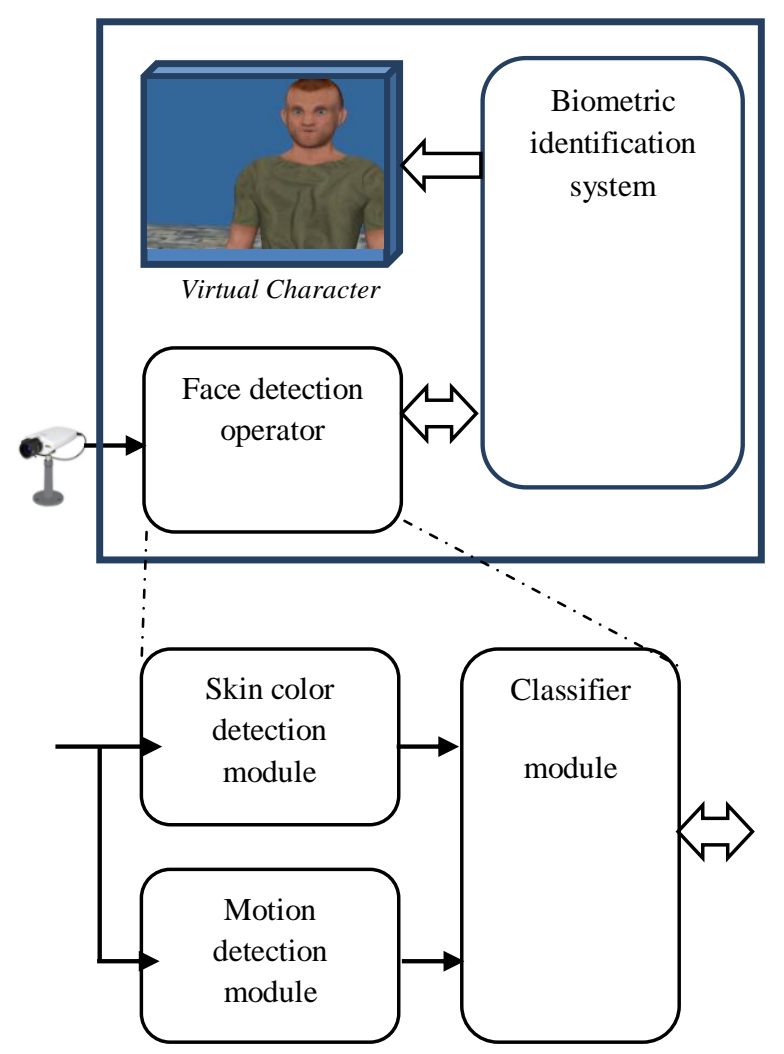

Fig 8: Interactive multimodal biometric identification system

The bloc diagrams of the different modules as well as theirs functional simulations are presented in figures 9, 10, 11, 12, 13 and 14. The parallelization of the research task increases considerably the speed of the detection even further at a cost of additional memory resources ( 8 memory 1 bit plans instead of one). Preliminary implementation results within a Virtex II pro FPGA Board [17] are summarized in table 1.

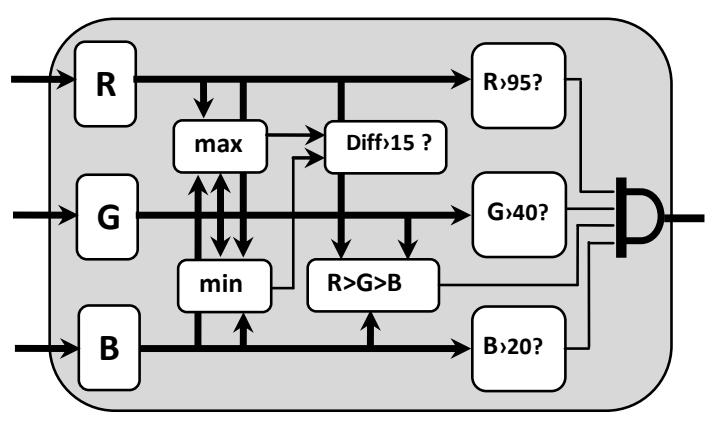

Fig 9: Skin color detection module

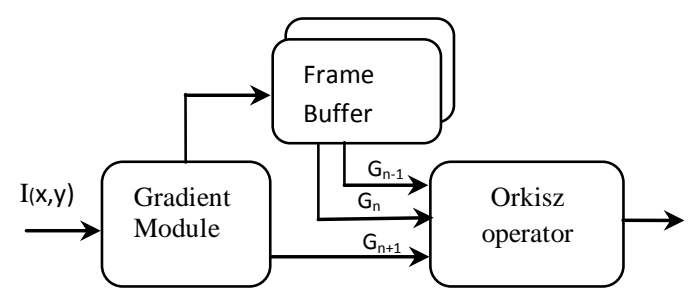

Fig 10: Motion detection module

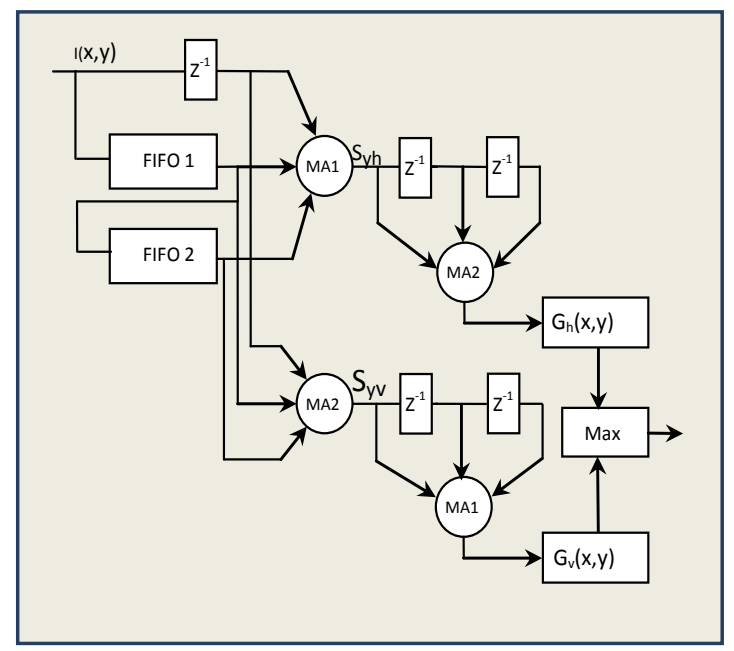

Fig 11: Gradient module

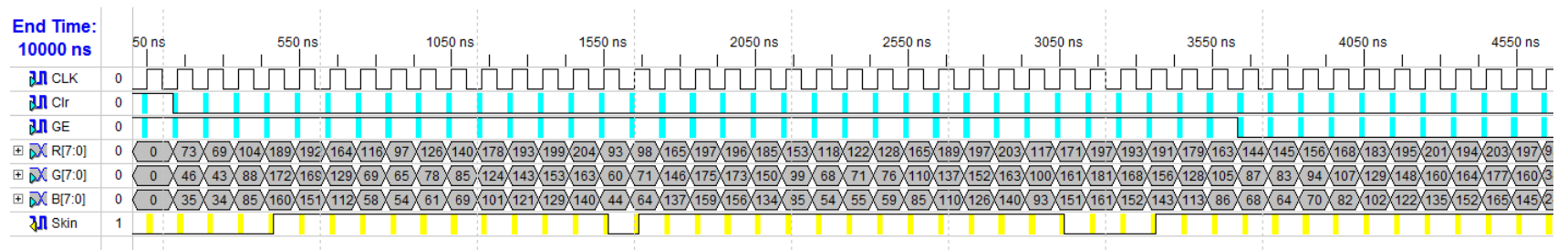

Fig 12: Functional simulation of the skin color detection module 


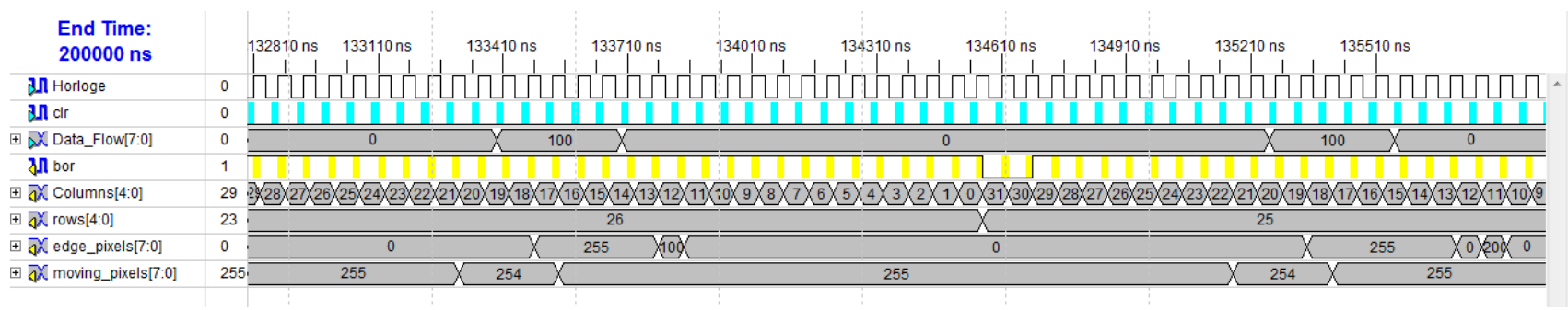

Fig 13: Functional simulation of the motion detection module

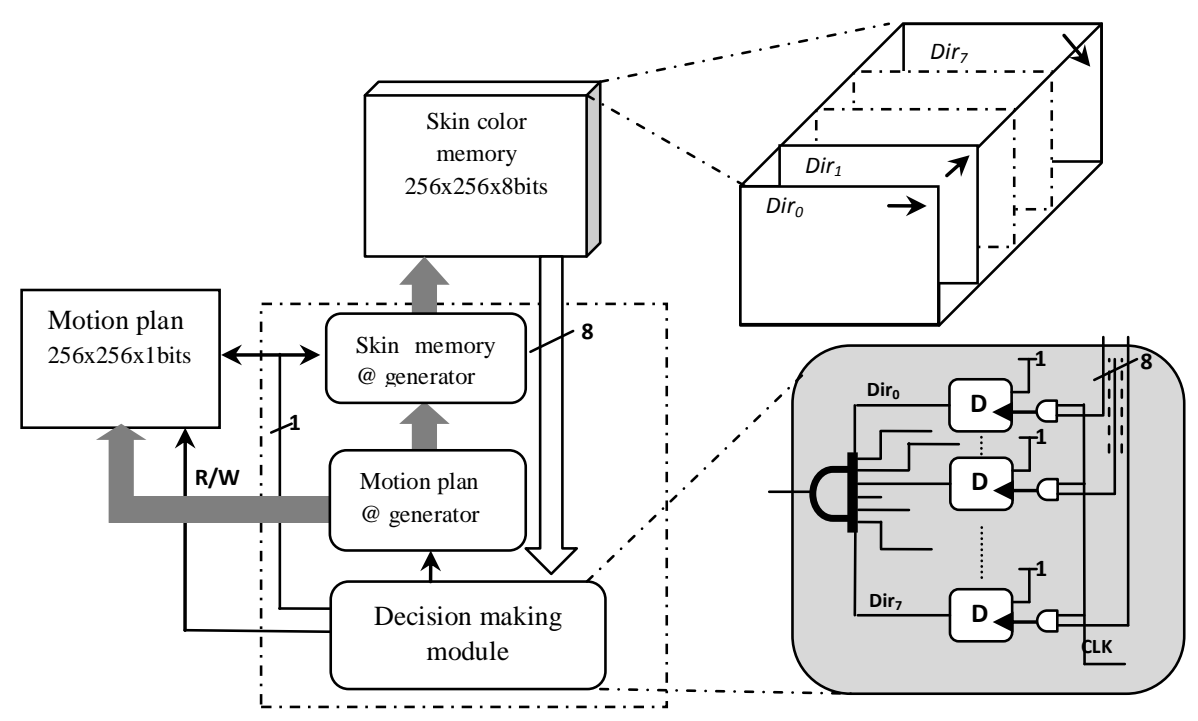

Fig 14: Classifier module

Table 1. Hardware implementation costs

\begin{tabular}{|c|c|c|c|}
\hline \multicolumn{4}{|c|}{ Motion detection module Utilization Summary } \\
\hline Logic Utilization & Used & Available & Utilization \\
\hline $\begin{array}{c}\text { Number of Slice } \\
\text { registers }\end{array}$ & 848 & 10,240 & $9 \%$ \\
\hline $\begin{array}{c}\text { Number of 4 inputs } \\
\text { LUTs }\end{array}$ & 1007 & 10.240 & $10 \%$ \\
\hline $\begin{array}{c}\text { Number of occupied } \\
\text { slices }\end{array}$ & 1833 & 5.120 & $36 \%$ \\
\hline
\end{tabular}

\section{CONCLUSION}

This work presents the development and the implementation of a dynamic face detection operator based on motion and color modalities. The different tests performed under controlled lighting have allowed us to validate the approach. An important work remains to be carried out in the Human/machine communication field to exploit efficiently the developed operator while regulating by conversation blocked cases due to: conflict situations (case of several people speaking at the same time), bad localization (person far from or near the camera), nonhuman dynamic forms etc .....

Future improvement will also focus on color spaces, with the aim to have a dynamic and adaptive color space choice, allowing thus robust face detection.

\section{ACKNOWLEDGMENTS}

This work was supported by the Algerian Research Ministry through the PNR project (Ref:42/TIC/2011). The hardware Verification tools are supported by the MSE Laboratory.

\section{REFERENCES}

[1] Martinez-Gonzalez, A. N. Ayala, R. V. 2011. Real-time face detection Using Neural Networks. Mexican International Conference on Artificial Intelligence. 2011. 144-149.

[2] Zhang, J. Zhang, X. D. Ha, S.W. 2008. A novel approach Using SVM and PCA for Face detection. Fourth International conference on Natural computation, ICNC '08. 29-33.

[3] Kicheon, H. Jihong, M. Wonchan, L. Jungchul, K. 2005. Real time face detection and recognition System using Haar-like feature HMM in Ubiquitous Network Environment. LNCS 3480.1154-1161.

[4] Lei, Z. Wang, C. Wang, Q. Huang, Y.2009. Real-Time Face Detection and Recognition for Video Surveillance Applications. World Congress on Computer Science and Information Engineering. WRI 2009.168-172.

[5] Khalifa, A. Sundaraj, K. Ibrahim, Z. Retnasamy, V. 2007. Complex Background Subtraction for Biometric identification. International Conference on Intelligent and Advanced Systems. ICIAS 2007. 696-701. 
[6] Viola, P. Jones, M.2004. Robust real-time face detection. International Journal of computer vision. Vol 57. 137154.

[7] Berkin, B. Berthold, K. P. Ichiro, M. 2010. Efficient Integral Image computation on the GPU. IEEE Intelligent Vehicle Symposium. 2010. 528- 532.

[8] Mustafah,Y. Bigdeli, A. Azman, A. Lovell, B. 2010. Square Patch Feature Based Face detection Architecture for high resolution smart camera. ACM/IEEE International Conference on distributed smart cameras. ICDSC 2010.175-181.

[9] Sadri, M. S. Shams, N. Rahmaty, M. Hosseini, I. Changiz, R. Mortazavian, S. Kheradmand, S.Jafari, R. 2004. An FPGA Based Fast Face Detector. Global Signal Processing Expo and Conference.

[10] Hiromoto, M. Nakahara, K. Sugano, H. Nakamura, Y. Miyamoto, R. 2007. A specialized processor suitable for AdaBoost-based detection with haar-like features. IEEE International conference on computer vision and pattern recognition, CVPR'07. 1-8.

[11] Hori, Y. Kuroda, T. 2007. A 0.79-mm2 29-mw real-time face detection core. IEEE Journal of Solid State Circuits, Vol. 42. 790-797.

\section{AUTHORS PROFILE}

Abbadi Belkacem received his Master degree in Electrical engineering in 2009. He is currently pursuing his $\mathrm{PhD}$ in reconfigurable architectures for image processing at MSE Laboratory, University of BBA, Algeria. His research interests include real time image processing and Biometry.

Adel Oulefki received his Master degree in Electrical engineering in 2009. He is currently pursuing his $\mathrm{PhD}$ in
[12] Wang, G. Yang, Y. Hue, K. 2010. A robust Facial Feature method based on optical flow and prior measurement. International Journal of Cognitive Informatics and neural intelligence. 62-75.

[13] Orkisz, M. 1992. Localisation d'objets mobiles dans des scènes filmées par une caméra fixe. Revue TSI.Vol 9. $\mathrm{N}^{\circ} 4$. 325-346.

[14] Menser, B. Wien, M. 2000. Segmentation and tracking of facial regions in color image sequences. SPIE Visual communication and Image Processing, Perth, Australia.

[15] Hsu, R. L. Abdelmottaleb, M. Jain, A. K. 2002. Face detection in color images. IEEE trans. Pattern Analysis and Machine Intelligence. 24(5). 696-706.

[16] Hartmut, F. W. Sadrozinski. Jinyuan, W. 2010. Applications of Field-Programmable Gate Arrays in Scientific Research. Taylor \& Francis. ISBN 978-14398-4133-4

[17] Xilinx Inc. Virtex II pro Platform FPGA User Guide.www.xilinx.com

image processing and classification at MSE Laboratory, University of BBA, ALGERIA. His current research involves real time pattern recognition and reconfigurable architectures. Messaoud Mostefai received his $\mathrm{PhD}$ in Automatic and Digital Signal Processing from the University of Reims (France) in 1995. His research interests include flexible and real time embedded systems, digital signal processing and video coding algorithms. 